\title{
Black holes, Boyle's Law and the quark-gluon plasma
}

\section{Brian P. Dolan*}

Maynooth University, Maynooth, Ireland and

Dublin Institute for Advanced Studies, 10 Burlington Rd, Dublin, Ireland

E-mail: bdolanethphys.nuim.ie

The role of the cosmological constant as a thermodynamic variable in black hole thermodynamics is discussed. A black hole in asymptotically anti-de Sitter space-time has a positive thermodynamic pressure associated with the cosmological constant and the conjugate thermodynamic variable is a volume. Isotherms in the $P-V$ plane can be constructed and there can be phase transitions between different black hole phases with critical points. Applications to the thermodynamics of conformal field theories are discussed, via the AdS/CFT correspondence. In particular critical exponents are calculated for the deconfining phase transition in $\mathscr{N}=$ supersymmetric $S U(N)$ Yang-Mills theory, in the limit of large $N$, and found to be mean field in this limit.

Proceedings of the Corfu Summer Institute 2015 "School and Workshops on Elementary Particle Physics and Gravity"

1-27 September 2015

Corfu, Greece

* Speaker. 


\section{Review of black hole thermodynamics}

Bekenstein suggested in 1972 that black holes should have an intrinsic entropy, proportional to the area of the event horizon, in Planck units [] and 2 years later Hawking succeeding in determining the associated temperature []. For a Schwarzschild black hole in 4-dimensional space-time, with line element

$$
d s^{2}=-f(r) d t^{2}+\frac{1}{f(r)} d r^{2}+r^{2} d \Omega^{2}
$$

where $f(r)=1-\frac{2 G M}{r}$, for example, the event horizon resides at $f\left(r_{h}\right)=0 \quad \Rightarrow \quad r_{h}=2 G M$ (we use units with $c=1$ ). The area of the event horizon is thus $A=16 \pi G^{2} M^{2}$ and the Bekenstein entropy is

$$
S \propto \frac{A^{2}}{\ell} P l,
$$

where $\ell_{P l}^{2}=\hbar G$. The surface gravity of the black hole is $\kappa=\frac{1}{4 G M}$ and the Hawking formula for the temperature is

$$
T=\frac{\kappa \hbar}{2 \pi}
$$

leading to

$$
T=\frac{\hbar}{8 \pi G M} \text {. }
$$

While this is completely negligible for astrophysical black holes, e.g. for a solar mass black hole $T=6 \times 10^{-8} \mathrm{~K}$, it is nevertheless very important for the conceptual understanding of black holes.

Identifying the mass of the black hole with the thermodynamic internal energy, a function of entropy, $M=U(S)$, we have

$$
T=\frac{\partial U}{\partial S}
$$

leading to the first law of black hole thermodynamics

$$
d M=T d S .
$$

With $S \propto \frac{A}{\hbar G}=\frac{16 \pi G M^{2}}{\hbar}$ and $T=\frac{\hbar}{8 \pi G M}$ this allows the constant of proportionality in (1.2) to be calculated giving the Hawking formula

$$
S=\frac{1}{4} \frac{A}{\hbar G} .
$$

More generally, when angular momentum and angular momentum $J$ and electric charge $Q$ are included, the first law reads

$$
d M=T d S+\Omega d J+\Phi d Q .
$$

\section{Smarr relation}

A consistency check of the first law is provided by combining Legendre transforms with dimensional analysis. In ordinary thermodynamics in $d$ dimensions $U\left(S, V, n_{i}\right)$ is a function of extensive variables: $S, V$ and the number of moles $n_{i}$, and $U$ itself is extensive. Extensive variables scale homogeneously as the system size is changed,

$$
\lambda^{d} U(S, V, n)=U\left(\lambda^{d} S, \lambda^{d} V, \lambda^{d} n_{i}\right) .
$$


Differentiating this with respect to $\lambda$ and then setting $\lambda=1$ gives the Euler relation

$$
\begin{aligned}
U & =S \frac{\partial U}{\partial S}+V \frac{\partial U}{\partial V}+n_{i} \frac{\partial U}{\partial n_{i}} \\
\Rightarrow \quad U & =S T-V P+n_{i} \mu_{i},
\end{aligned}
$$

where $\mu_{i}$ is the chemical potential. This allows us to write the Gibbs free energy, the Legendre transform of $U$ with respect to $S$ and $V$, as

$$
G\left(T, P, n_{i}\right)=U+V P-S T=n_{i} \mu_{i}
$$

which is an integrated version of the Gibbs-Duhem relation.

A similar analysis can be applied to a rotating black hole in $D$ space-time dimensions. Converting masses to lengths using Newton's constant, and subsequently setting $G=1$, the mass, entropy and angular momentum scale as

$$
M \rightarrow \lambda^{D-3} M, \quad S \rightarrow \lambda^{D-2} S, \quad \mathbf{J} \rightarrow \lambda^{D-2} \mathbf{J}
$$

(only neutral black holes are considered in this section, charged black holes will be considered adding a charge does not significantly change the conclusions). The black hole mass is a function of $S$ and $\mathbf{J}$ so

$$
\begin{aligned}
& \lambda^{D-3} M(S, J)=M\left(\lambda^{D-2} S, \lambda^{D-2} \mathbf{J}\right) \\
& \Rightarrow(D-3) M=(D-2) S \frac{\partial M}{\partial S}+(D-2) \mathbf{J} \cdot \frac{\partial M}{\partial \mathbf{J}}
\end{aligned}
$$

leading to the Smarr relation []

$$
(D-3) M=(D-2) S T+(D-2) \mathbf{J} . \Omega
$$

which is satisfied for all known rotating black solutions of Einstein's equations in $D$ space-time dimensions which are asymptotically flat.

However the naïve Smarr relation above fails in asymptotically AdS space-time. The reason for this is clear - the cosmological constant is another dimensionful parameter that must be included in the dimensional analysis. Following Henneaux and Teitelboim [] we define a Legendre transform for $\Lambda$

$$
\Theta:=\frac{\partial M}{\partial \Lambda} .
$$

The cosmological constant has length dimension -2 , so rescaling lengths results in $\Lambda \rightarrow \lambda^{-2} \Lambda$ and

$$
\begin{aligned}
\lambda^{D-3} M(S, \Lambda, \mathbf{J}) & =M\left(\lambda^{D-2} S, \lambda^{-2} \Lambda, \lambda^{D-2} \mathbf{J}\right) \\
\Rightarrow \quad(D-3) M & =(D-2) S T-2 \Theta \Lambda+(D-2) \mathbf{J} . \Omega,
\end{aligned}
$$

which is the correct modification of (2.6) in asymptotically de Sitter or anti-de Sitter space-time, as first observed in []. 


\section{Pressure and enthalpy}

A cosmological constant $\Lambda$ contributes a pressure $P$ and energy density $\varepsilon$ with equation of state

$$
\varepsilon=-P=\frac{\Lambda}{8 \pi} \text {. }
$$

It should be expected that the energy density in a volume $V$ will contribute to the thermal energy, which is a function of $S$ and $V, U(S, V)$,

$$
U=M+\varepsilon V=M-P V
$$

implying that

$$
M=U+P V
$$

is the Legendre Legendre transform $U(S, V)$. The thermodynamic potential with this property is the enthalpy $H(S, P)$, a function of $S$ and $P$,

$$
M=U+P V=H(S, P),
$$

as first suggested in []. Within this framework we define the volume to be the thermodynamically conjugate variable to the pressure

$$
V=\left.\frac{\partial M}{\partial P}\right|_{S}
$$

and the first law becomes []

$$
d U=T d S-P d V
$$

\subsection{Critical behaviour of asymptotically AdS Kerr black holes}

Isotherms can be plotted in the $P-V$ plane and it transpires that black hole solution of Einstein's equations often behave remarkably like van der Waals gases, with a line of first order phase transitions between 'large' and 'small' black holes, terminating at a critical point.

For example an asymptotically AdS Kerr black hole has a second order phase transition [] and this is visible in the $P-V$ diagram in figure 1 . The line of phase transitions is clearly visible in the phase diagram in the $P-T$ plane in figure 2.

There is a latent heat across the line of phase transition,

$$
L=T \Delta S=M_{\text {large }}-M_{\text {small }}
$$

and one can check that the Clapeyron equation is satisfied []

$$
\frac{d P}{d T}=\frac{\Delta S}{\Delta V}
$$

The critical point is in fact in exactly the same universality class as a van der Waals as it has mean field critical exponents, []. The internal energy is a function of $S, V$ and $J, U(S, V, J)$ and we can define reduced thermodynamic variables near the critical point,

$$
p=\frac{P-P_{\text {crit }}}{P_{\text {crit }}}, \quad v=\frac{V-V_{\text {crit }}}{V_{\text {crit }}}, \quad t=\frac{T-T_{\text {crit }}}{T_{\text {crit }}} .
$$




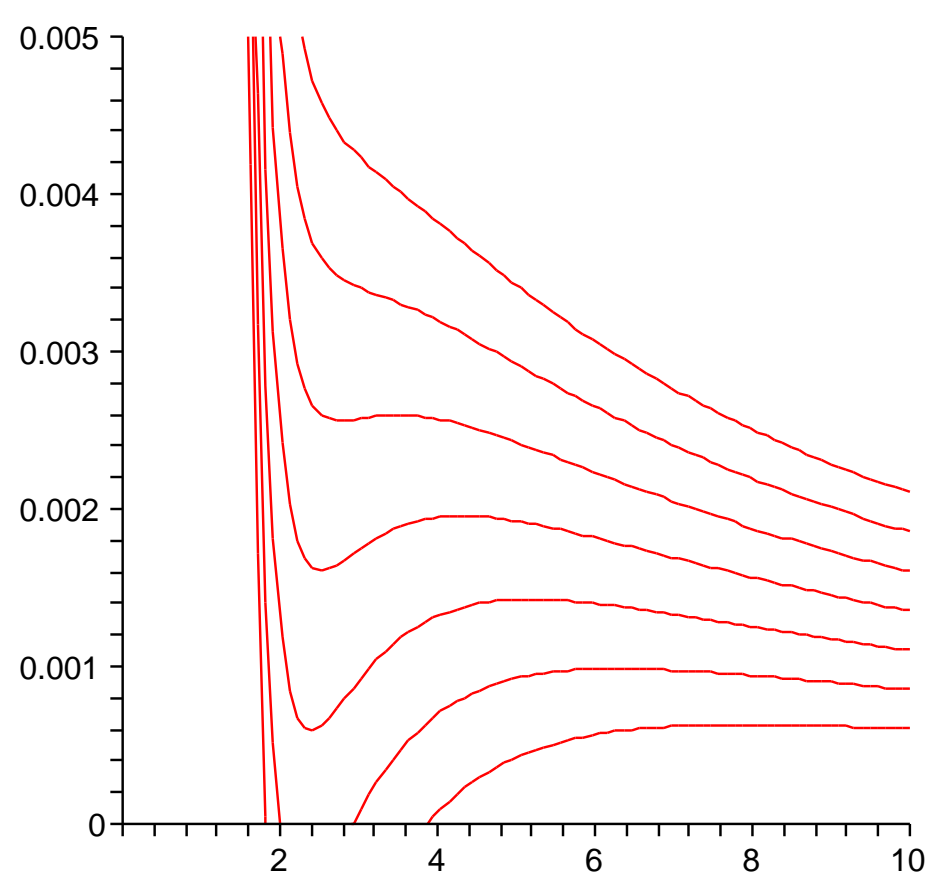

Figure 1: Isotherms, in the $P-V$ plane, for an asymptotically AdS Kerr black hole rotating with angular momentum $J$. Van der Walls type behaviour is clearly visible. (What is actually plotted is $P J$ versus $V^{\frac{1}{3}} / J^{\frac{1}{2}}$ at fixed $J$, which are dimensionless variables).

The critical exponents are define as usual:

- for the heat capacity

$$
C_{V}=\left.T \frac{\partial T}{\partial S}\right|_{V, J} \propto|t|^{-\alpha}
$$

- at fixed $p<0$ the jump in volume between large black holes, with reduced volume $v_{>}$, and small black holes, with reduced volume $v_{<}$, is

$$
\Delta v=v_{>}-v_{<} \propto|t|^{\beta}
$$

- for the isothermal compressibility

$$
-V\left(\frac{\partial P}{\partial V}\right)_{T, J} \propto|t|^{-\gamma}
$$

- on the critical isotherm, when $t=0$,

$$
|p| \propto|v|^{\delta}
$$

The critical exponents for an asymptotically AdS Kerr black hole have been calculated [] and they are mean field

$$
\alpha=0, \quad \beta=\frac{1}{2}, \quad \gamma=1 \quad \text { and } \quad \delta=3 .
$$




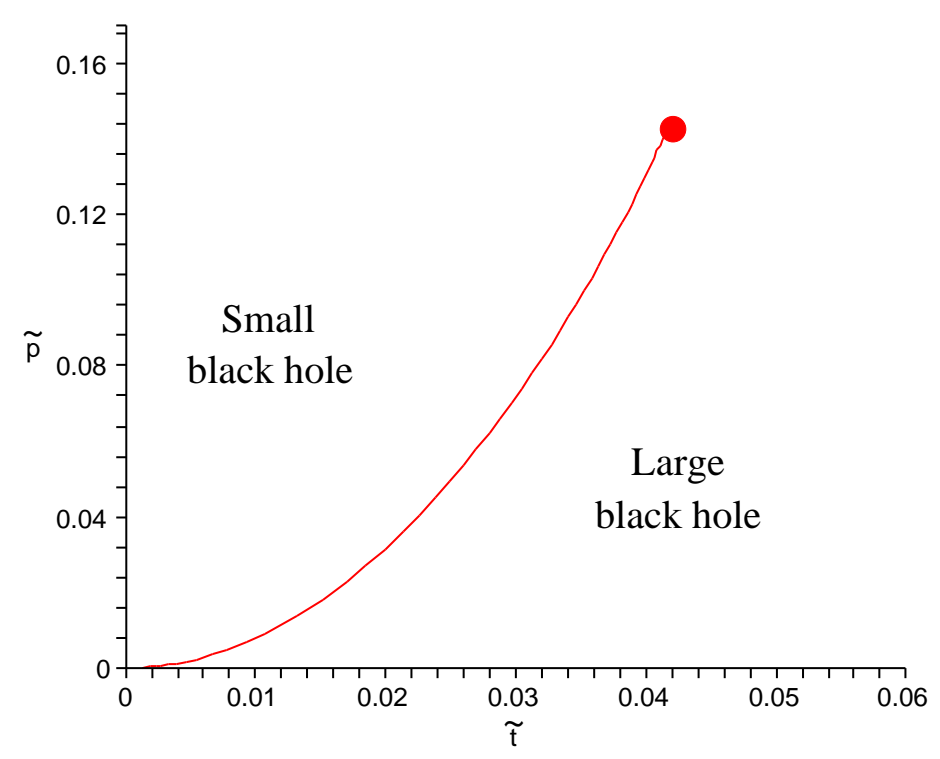

Figure 2: Phase diagram, in the $P-T$ plane, for an asymptotically AdS Kerr black hole rotating with angular momentum $J$ (in dimensionless variables). There is a line of first order phase transitions separating two black hole phases, 'large' black holes and 'small' black holes, which coalesce at a critical point.

\section{Higher dimensional black holes}

In space-time dimensions greater than 4 there can be more than one angular variable characterising a rotating black hole. In 6-dimensions, for example, the isometry group of a spherical event horizon is the rank 2 group $S O(5)$ and there are two angular momenta, $J_{1}$ and $J_{2}$. It was shown in [] that the phase diagram is more complicated than the 4-dimensional case - it depends on the ratio $q=\frac{J_{1}}{J_{2}}$ and has three phases: corresponding to large, small and intermediate size black holes. There are three lines of first order phase transitions meeting at a triple point, two of which terminate at critical points.

While there are examples of higher dimensional black holes that have critical points with exponents that are not mean field, [], these are not solutions of simple Einstein gravity with a cosmological constant, they involve modified gravitational dynamics such as Lovelock gravity or Einsein-Gauss-Bonnet gravity, with extra dimensionful parameters.

\section{AdS/CFT}

Maldacena has conjectured an equivalence between gravity and conformal field theories (CFT's) [] in which the CFT lives on the asymptotic boundary of anti-de Sitter space-time. In this scenario weak, classical, gravity in the bulk corresponds to strongly coupled CFT on the boundary, in a space-time of one dimension lower than the gravitational dynamics.

For example for $10-d$ superstring theory compactified on $A d S_{5} \times S^{5}$ the cosmological constant in $A d S_{5}$ is related to the radius $L$ of $S^{5}$ by $\Lambda=-\frac{4}{L^{2}}$. The CFT on the boundary is $\mathscr{N}=4$ supersymmetric Yang-Mills with gauge group $S U(N)$ in 4 space-time dimensions. The number of 
colors is determined by $L$ and the Planck length of 10-dimensional supergravity, $\ell_{P l}^{8}=\hbar G_{10}$, by []

$$
N=\frac{\pi^{2} L^{4}}{\sqrt{2} \ell_{P l}^{4}} .
$$

If $G_{10}$ is fixed then varying $\Lambda$ (or equivalently $L$ ) necessitates varying the number of colors and it was suggested in [] that the variable thermodynamically conjugate to $\Lambda$ in this context would be a kind of chemical potential for color. This point of view was developed more quantitatively in [].

Here we shall adopt a different approach and keep $N$ fixed as $L$ is varied. This requires varying $G_{10}$ as $L$ is varied so as to keep the right hand side of (5.1) fixed, as suggested in []. From the point of view of the full string theory the 10-dimensional Newton constant is related to the string tension $\alpha^{\prime}$ and the string coupling $g_{s}$ by $\hbar G_{10}=8 \pi^{6} g_{s}^{2}\left(\alpha^{\prime}\right)^{4}$, so varying $G_{10}$ can be viewed as varying $g_{s}$ keeping $\alpha^{\prime}$ fixed.

Following [] we fix $N$ and vary $\Lambda$. According to the AdS/CFT conjecture the strong coupling limit of the SUSY Yang-Mills theory is related to weak (classical) gravity in the 5-dimensional bulk, in particular an asymptotically AdS black hole in 5-dimensions translates to a finite temperature thermal background in the 4-dimensional Yang-Mills theory on the boundary. Including a $U(1)$ charge $q$ on the black-hole the 5-dimensional line element, in global co-ordinates, is []

$$
d^{2} s=-f(r) d t^{2}+\frac{d r^{2}}{f(r)}+r^{2} d^{2} \Omega_{3}
$$

with

$$
f(r)=1-\frac{\mu}{r^{2}}+\frac{q^{2}}{r^{4}}+\frac{r^{2}}{L^{2}}
$$

where $d^{2} \Omega_{3}$ is the line element on $S^{3}$ with unit radius. The asymptotic boundary of $A d S_{5}$ at $r \rightarrow \infty$ has the topology of $R \times S^{3}$, with constant time slices being 3-spheres of volume $V=2 \pi^{2} L^{3}$. Varying $L$ in this solution is equivalent to varying the volume of the $S^{3}$ slices.

The usual mass $M$ (the AdS equivalent of the ADM mass) of the black hole are related to the metric parameter, $\mu$, by

$$
M=\frac{3 \pi \mu}{8 G_{5}},
$$

and, in terms of the largest root $r_{h}$ of $f(r)=0$,

$$
M=\frac{3 \pi r_{h}^{2}}{8 G_{5}}\left(1+\frac{r_{h}^{2}}{L^{2}}+\frac{q^{2}}{r_{h}^{4}}\right) .
$$

The 5-dimensional Newton's constant $G_{5}$ is related to $G_{10}$ by the volume of the 5-sphere in the $A d S_{5} \times S^{5}$ solution. The 5 -sphere has radius $L$, and volume $\pi^{3} L^{5}$, leading to

$$
\frac{\pi^{3} L^{5}}{\hbar G_{10}}=\frac{1}{\hbar G_{5}} \quad \Rightarrow \quad \frac{1}{\hbar G_{5}}=\frac{2 N^{2}}{\pi L^{3}}
$$

As written the 5-dimensional charge, $q$, has dimensions of length squared but the charge, $Q$, in the CFT should be dimensionless, as appropriate to a charge in 4-dimensions. A dimensionless 4-d charge can therefore defined as []

$$
Q=\frac{\sqrt{3} \pi L}{2 \hbar G_{5}} q=\sqrt{3} N^{2} \frac{q}{L^{2}}
$$


(the $3 \pi$ follows from Gauss' law and the convention for the normalisation of the $F^{2}$ term in the action, actually $-\frac{1}{16 \pi G_{5}} F^{2}$, but its explicit value does not really matter in the sequel).

Finally the event horizon has the topology of $S^{3}$, with a radius $r_{h}$, and the entropy is $\frac{1}{4}$ of the area (or 3-dimensional volume in this case). In 5-d Planck units,

$$
S=\frac{1}{4}\left(\frac{2 \pi^{2} r_{h}^{3}}{\hbar G_{5}}\right)=\pi N^{2}\left(\frac{r_{h}}{L}\right)^{3}
$$

\subsection{Quark-gluon plasma}

At high temperature the Yang-Mills theory on the boundary will be in the deconfined phase []. Translating from the geometric variables $\left(r_{h}, L, q\right)$ to the thermodynamic variables $(S, V, Q)$ the mass (5.5) is equated to the internal energy,

$$
M\left(r_{h}, L, q\right)=U(S, V, Q)=\frac{3 N^{2} \hbar}{4}\left(\frac{2 \pi^{2}}{V}\right)^{\frac{1}{3}}\left(x^{4}+x^{2}+\frac{y^{2}}{x^{2}}\right)
$$

where $x=\left(\frac{S}{\pi N^{2}}\right)^{\frac{1}{3}}=\frac{r_{h}}{L}$ and $y=\frac{Q}{\sqrt{3} N^{2}}=\frac{q}{L^{2}}$ are dimensionless.

Note that in the CFT the black hole mass is equated to the internal energy of the system while in the bulk $A d S_{5}$ it is interpreted as the enthalpy. This because $\Lambda$ relates to a volume in the CFT while it is a pressure on the gravitational side. This has the effect of interchanging $P$ and $V$ in the CFT relative to gravity in the bulk.

The temperature, pressure and electrostatic potential follow from differentiation of (5.9),

$$
T=\left.\frac{\partial U}{\partial S}\right|_{V, Q}, \quad P=-\left.\frac{\partial U}{\partial V}\right|_{S, Q}, \quad \Phi=\left.\frac{\partial U}{\partial Q}\right|_{S, V},
$$

and are found to be

$$
\begin{gathered}
T=\left.\frac{\partial U}{\partial S}\right|_{Q, V}=\frac{\hbar}{2 \pi L}\left(2 x+\frac{1}{x}-\frac{y^{2}}{x^{5}}\right), \\
P=-\left.\frac{\partial M}{\partial V}\right|_{S, Q}=\frac{N^{2} \hbar}{8 \pi^{2} L^{4}}\left(x^{4}+x^{2}+\frac{y^{2}}{x^{2}}\right)=\frac{\varepsilon}{3},
\end{gathered}
$$

where $\varepsilon=\frac{U}{V}$ is the energy density, and

$$
\Phi=\left.\frac{\partial U}{\partial Q}\right|_{S, V}=\frac{\sqrt{3} \hbar}{2 L} \frac{y}{x^{2}} .
$$

The heat capacity, $C_{V, Q}=\left.T \frac{\partial U}{\partial T}\right|_{V, Q}$, is found to be positive and diverges at a critical point, first found by []. This is most succinctly parametrized by the critical values of $x$ and $y$

$$
x_{*}^{2}=\frac{1}{3}, \quad y_{*}^{2}=\frac{1}{135} .
$$

For $Q=0$ there is a first order phase transition, which corresponds to the Hawking-Page phase transition in bulk [], and this is translates to the deconfining phase transition for QCD on the boundary []. This extends for non-zero $Q$ to a line of first order phase transitions terminating at the critical 

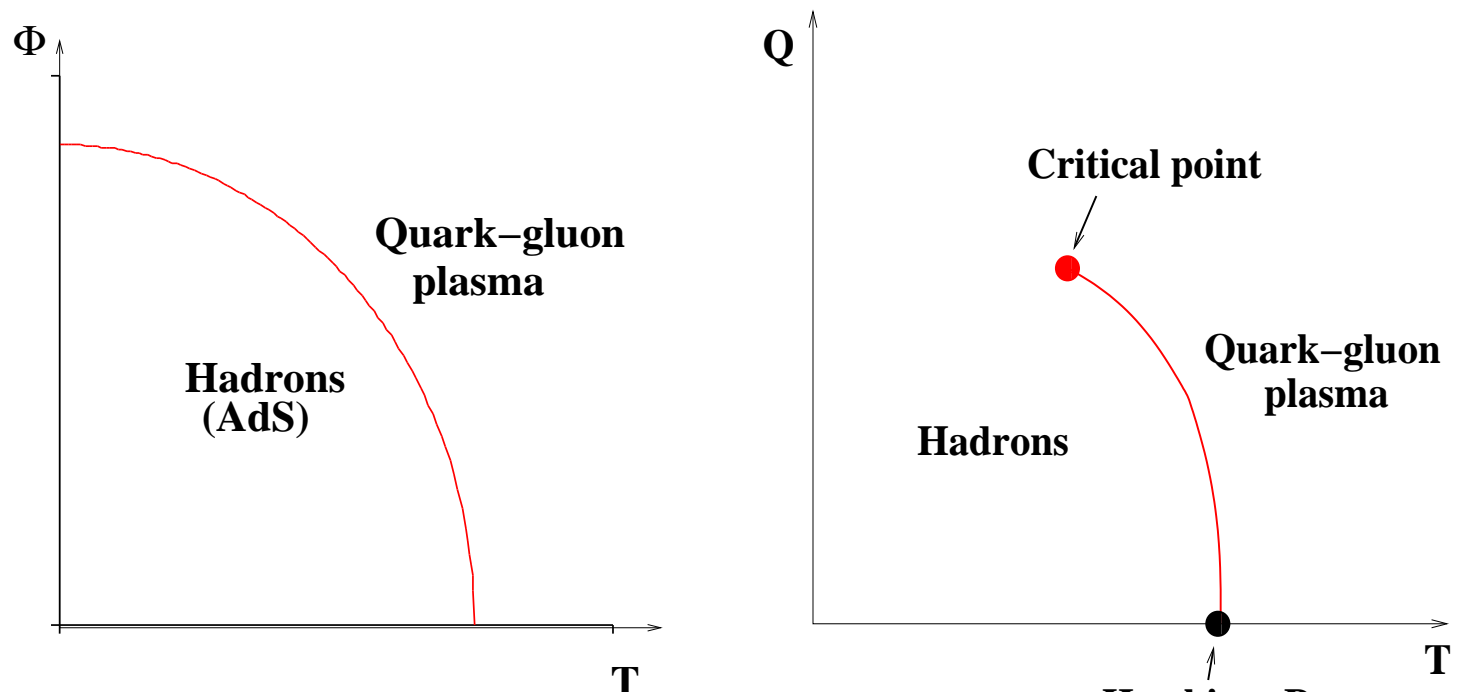

Hawking-Page phase transition

Figure 3: Phase diagrams for the boundary CFT in the $\Phi-T$ and $Q-T$ planes.

point. This phase structure was found in [] and the phase diagrams in the $\Phi-T$ plane and the $Q-T$ planes were worked out there, they are sketched in figure 3 .

With the pressure defined as above one can go further and determine the adiabatic compressibility

$$
\kappa_{S, Q}=-\left.\frac{1}{V} \frac{\partial V}{\partial P}\right|_{S, Q}=\frac{3}{4 P}
$$

which is positive and finite - there is no sign of the critical point in the adiabatic compressibility. Critical behaviour is however manifest in the isothermal compressibility,

$$
\kappa_{T, Q}=-\left.\frac{1}{V} \frac{\partial V}{\partial P}\right|_{T, Q},
$$

which vanishes at the critical point! This a reflection of the fact that $P$ and $V$ in the CFT are swapped round from their roles in the gravitational bulk, and it is the inverse of the isothermal compressibility that diverges in the CFT.

\subsection{Critical exponents in the $P-V$ plane}

Define the reduced temperature

$$
t=\frac{T-T_{*}}{T_{*}}
$$

evaluated at fixed $V$ ( $L$ cancels in the ratio $t$ and plays no role in $C_{V, Q}$ ). One finds that the heat capacity diverges at the critical point as

$$
C_{V, Q} \sim|t|^{-\alpha}
$$

with $\alpha=\frac{2}{3}$.

At first sight this is a little surprising as the critical behaviour of charged black holes was examined in [] and found to be mean field, and mean field exponents would give $\alpha=0$. 
To understand what is happening here it is useful to use dimensionless variables for the pressure and the volume. To study isothermal properties one might use

$$
\widetilde{V}:=V T^{3}=\frac{\hbar^{3}}{4 \pi}\left(\frac{2 x^{6}+x^{4}-y^{2}}{x^{5}}\right)^{3}
$$

and

$$
\widetilde{P}:=\frac{P}{N^{2} T^{4}}=\frac{2 \pi^{2} x^{18}}{\hbar^{3}} \frac{\left(x^{6}+x^{4}+y^{2}\right)}{\left(2 x^{6}+x^{4}-y^{2}\right)^{4}} .
$$

A plot of $\widetilde{P}$ against $\widetilde{V}$ for various values of $y$ is shown in figure 4 . The critical point is at $\widetilde{V}_{*}=$ $\frac{384 \sqrt{3}}{125 \pi} \approx 1.694, \widetilde{P}_{*}=\frac{875 \pi^{2}}{55296} \approx 0.156$.

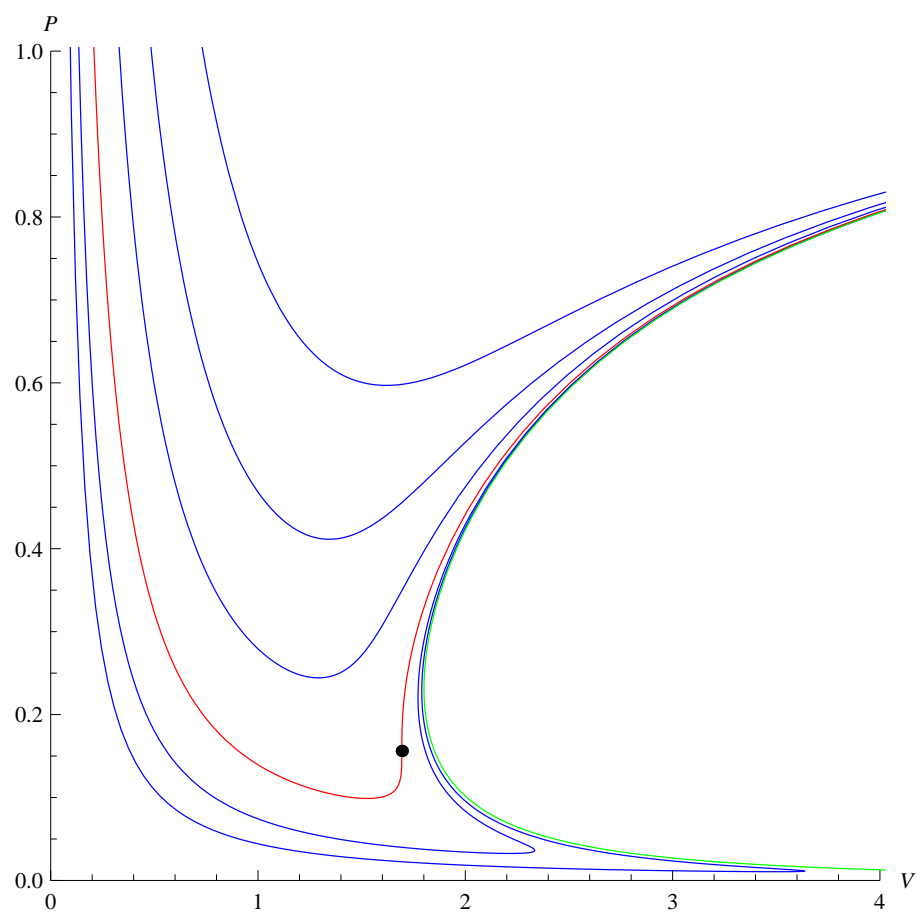

Figure 4: Curves of constant $Q$ in the $P-V$ plane $\left(P / T^{4}\right.$ plotted against $\left.V T^{3}\right)$. The critical curve is red and the critical point is indicated by a black dot. The green curve is $Q=0$.

We see that $\left.\frac{\partial \widetilde{P}}{\partial \widetilde{V}}\right|_{T, Q}$ diverges to $+\infty$ at the critical point so, as mentioned earlier, the isothermal compressibility (5.16) vanishes there. But if we are interchanging $P$ and $V$ relative to their roles in the bulk, the critical point should be expected to manifest itself as a divergence in the inverse isothermal compressibility, not in the compressibility itself, and this is indeed the case.

Normally other critical exponents follow by defining a reduced pressure and volume,

$$
p=\frac{\widetilde{P}-\widetilde{P}_{*}}{\widetilde{P}_{*}}, \quad v=\frac{\widetilde{V}-\widetilde{V}_{*}}{\widetilde{V}_{*}},
$$

but conformal invariance causes a complication here in that $t$ and $v$ are not independent, rather

$$
(1+v)=(1+t)^{3} .
$$


This is because the finite temperature theory on $S^{3}$ is equivalent to a theory with Euclidean time periodically identified which is topologically $S^{1} \times S^{3}$. In a conformally invariant theory the physics is only sensitive only to the ratio of the radii of $S^{1}$ to $S^{3}$ not to each of the radii separately. We can use either $t$ or $v$ to probe the physics, but not both.

Better is to fix one, let us choose $t$, and vary $v$. The curves in figure 4 are at fixed charge, so we shall use charge as the control parameter, rather than $t$, to explore the physics in the $P-V$ plane. We define the reduced charge $\eta$ as

$$
\eta=\left(y-y_{*}\right) / y_{*} .
$$

From figure 4 we see that, at a fixed charge in the two phase regime $y<y_{*}$ (to the right of the critical point), there is a jump in the pressure at a fixed volume. This implies that pressure should be viewed as the order parameter and, with $\eta$ as the control parameter, the exponent $\delta$ is then obtained from Maxwell's equal area law, applied to $v(p)$ rather than $p(v)$, at constant $Q$ and $T$. The jump in pressure is parametrized by

$$
\Delta p=p_{>}-p_{<} \propto|\eta|^{\beta} .
$$

With $p$ is the order parameter and $\eta$ as the control parameter $\alpha$ is obtained from the divergence of $C_{P, Q} \sim|\eta|^{-\alpha}$, which is actually finite at the critical point, and so $\alpha=0$.

The inverse isothermal compressibility

$$
\left(\kappa_{T, Q}\right)^{-1} \sim|\eta|^{-\gamma}
$$

does diverge, while on the critical curve $\eta=0$

$$
|v| \propto|p|^{\delta} .
$$

Explicit evaluation reveals that $\alpha, \beta, \gamma$ and $\delta$ are again mean field exponents

$$
\alpha=0, \quad \beta=\frac{1}{2}, \quad \gamma=1 \quad \text { and } \quad \delta=3 .
$$

\section{Conclusions}

It has been argued that, in gravitational theories with a variable cosmological constant, the black hole mass should identified with enthalpy,

$$
M=H=U+P V
$$

and not the more usual internal energy. This allows the definition of a "thermodynamic volume"

$$
V=\frac{\partial H}{\partial P}
$$

and $\Lambda=-8 \pi P \neq 0$ implies that there should be a $P d V$ term in the first law of black hole thermodynamics which is modified to read

$$
d U=T d S-P d V+\Omega d J+\Phi d Q .
$$


This $P d V$ term in the first law affects the efficiency of Penrose processes which can be up to 75\% efficient for a charged black hole in asymptotically AdS space-time, as opposed to 50\% for an asymptotically flat charged black hole [].

There are critical points in the black hole phase diagram and all known asymptotically AdS black hole solutions of Einstein's equations exhibit mean field exponents. Various well known thermodynamic relations, such as the Clapeyron equation, can be verified in black hole thermodynamics. The adiabatic compressibility of a black hole can be defined and in any dimension this is positive and non-singular $0 \leq \kappa_{S, P}<\infty$, at least for asymptotically AdS Myers-Perry black holes.

Within the framework of the AdS/CFT correspondence the thermodynamics of asymptotically AdS black holes in the bulk gives us information about the thermodynamic behaviour of the conformal field theory in the boundary. The mass there is identified with internal energy and, in global co-ordinates, the cosmological constant can be translated to a finite volume for the CFT. This in turn leads to the calculation of the pressure in the CFT $P=-\frac{\partial U}{\partial V}$.

For $A d S_{5} \times S^{5}$ compactification of 10-dimensional superstring theory a charged black hole in $A d S_{5}$ corresponds to $\mathscr{N}=4$ SUSY Yang-Mills in 4 space-time dimensions, with a spatial geometry which is $S^{3}$. There is a line of first order phase transitions, which terminates in a critical point which has mean field exponents, with pressure and volume interchanged from the usual van der Waals case.

Acknowledgment: This article is based upon work from COST Action MP1405 QSPACE, supported by COST (European Cooperation in Science and Technology).

\section{References}

[1] J.D. Bekenstein, Lett. Nuovo. Cimento 4 (1972) 737; J.D. Bekenstein, Phys. Rev. D7 (1973) 2333.

[2] S.W. Hawking, Nature 248 (1974) 30; S.W. Hawking, Comm. Math. Phys. 43 (1975) 199; S.W. Hawking, Phys. Rev. D13 (1976) 191.

[3] L. Smarr, Phys. Rev. Lett. 30, (1973) 71.

[4] M. Henneaux and C. Teitelboim, Phys. Lett. 143B, (1984) 415; ibid. 222B, (1989) 195.

[5] D. Kastor, S. Ray and J. Traschen, Class. Quantum Grav. 26, (2009) 195011, [arXiv:0904.2765].

[6] B.P. Dolan, Class. Quantum Grav. 28 (2011) 125020, [1008.5023].

[7] M.M. Caldarelli, G. Cognola and D. Klemm, Class. Quantum Grav. 17 (2000) 399, [hep-th/9908022].

[8] B.P. Dolan, Class. Quantum Grav. 28 (2011) 235017, [arXiv:1106.6260];

[9] D. Kubižnák and R.B. Mann, JHEP 1207 (2012) 033, [arXiv:1205.0559];

[10] B.P. Dolan Where is the PdV term in the first law of black hole thermodynamics? in Open Questions in Cosmology, ed. G.J. Olmo (2012) InTech [arXiv:1209.1272].

[11] B.P. Dolan, Phys. Rev. D90 (2014) 084002, [arXiv:1407.4037].

[12] N. Altamirano, D. Kubizn̆ák, R.B. Mann and Z. Sherkatghanad, Galaxies 2 (2014) 89, [arXiv:1401.2586]. 
[13] B.P. Dolan, A. Kostouki, D. Kubizn̆ák and R.B. Mann, Class. Quantum Grav. 31 (2014) 242001, [arXiv:1407.4783].

[14] J. Maldacena, Adv. Theor. Math. Phys. 2 (1998) 231, [hep-th/9711200].

[15] C.V. Johnson. Class. Quantum Grav. 31 (2014) 205002, [arXiv:1404.5982 [hep-th]].

[16] B.P. Dolan, JHEP 1410 (2014) 179, [arXiv:1406.7267].

[17] A. Karch and B. Robinson, JHEP 1512 (2015) 073, [arXiv:1510.02472].

[18] A. Chamblin, R. Emparan, C.V. Johnson and R.C. Myers, Phys. Rev. D 60 (1999) 064018, [hep-th/9902170].

[19] C. Niu, Y. Tian and X. Wu, Phys. Rev. D 85 (2012) 024017, [arXiv: 1104.3066].

[20] A. Chamblin, R. Emparan, C.V. Johnson and R.C. Myers, Phys. Rev. 60 (1999) 104026, [hep-th/9904197].

[21] S.W. Hawking and D.N. Page, Comm. Math. Phys. 87, (1983) 577.

[22] E. Witten, Adv. Theor. Math. Phys. 2 (1998) 253, [arXiv:hep-th/9802150]; ibid. 2 (1998) 505, [arXiv:hep-th/9803131]. 